\title{
Acceso a la información de mujeres con VPH, displasia y cáncer cervical in situ
}

\author{
Ma del Carmen Castro-Vásquez, D en C Pol y Soc,(') Ma del Carmen Arellano-Gálvez, M en C Soc.(I)
}

\begin{abstract}
Castro-Vásquez MC,Arellano-Gálvez MC. Acceso a la información de mujeres con VPH, displasia y cáncer cervical in situ. Salud Publica Mex 2010;52:207-2I 2.
\end{abstract}

\begin{abstract}
Resumen
Objetivo. Presentar un análisis relacional de cómo mujeres diagnosticadas con el virus del papiloma humano (VPH), displasia del cuello del útero o neoplasias del cuello uterino, reciben y/o acceden a la información y cómo la viven en sus relaciones cercanas. Material y métodos. En 2008 se realizaron 34 entrevistas cualitativas a mujeres en dos clínicas de colposcopía de la Secretaría de Salud, en Hermosillo, Sonora. El análisis se basó en la teoría fundamentada. Resultados. Existe una franca analogía entre cáncer cervicouterino $(\mathrm{CaCu})$ y muerte, una amplia desinformación sobre VPH y displasias y una práctica persistente entre los médicos de no ofrecer información oportuna y clara a las pacientes. Existe una apreciación estigmatizante hacia la infección por VPH que afecta las relaciones cercanas de las mujeres. Conclusión. A pesar de la necesidad de las pacientes de obtener información, no la exigen al médico, lo que contribuye a su desconfianza y angustia.
\end{abstract}

Palabras clave: virus del papiloma humano; displasia del cuello del útero; neoplasias del cuello uterino; acceso a la información; México
Castro-Vásquez MC,Arellano-Gálvez MC.

Access to information by women with HPV, cervical dysplasia and cancer in situ.

Salud Publica Mex 2010;52:207-212.

\section{Abstract}

Objective. To present a relational analysis of how women who are diagnosed with the human papilloma virus (HPV), cervical dysplasia or cervical neoplasia receive or seek information, and how they experience this process within their immediate relationships. Materials and Methods. In 2008, 34 qualitative interviews were carried out with women at two Secretary of Health colposcopy clinics in Hermosillo, Sonora. Analysis was based on grounded theory. Results. There is a patent analogy between cervical cancer and death, much disinformation about HPV and dysplasias, and a persistent lack of timely and clear information given to patients by doctors. There is a stigma attached to HPV infection which affects women's immediate relationships. Conclusion. Despite patients' need to obtain information, they do not demand it from their doctor, which contributes to their anguish and distrust.

Key words: HPV; cervical dysplasia; cervical neoplasia; access to information; Mexico
$E^{1}$ virus del papiloma humano (VPH) es una de las infecciones de trasmisión sexual más frecuentes en el mundo, misma que puede ser asintomática por largos periodos de tiempo. ${ }^{1,2}$ Los reportes epidemiológicos señalan que el VPH se presenta en $99.7 \%$ de los casos positivos de cáncer cervicouterino $(\mathrm{CaCu})$, y en estudios nacionales se ha reportado que la presencia de VPH de alto riesgo aumenta 78 veces la probabilidad de presentar $\mathrm{CaCu}^{3,4} \mathrm{El}$ riesgo y la sintomatología del VPH son distintos entre hombres, en los que generalmente

(I) Centro de Estudios en Salud y Sociedad (CESS), El Colegio de Sonora. Sonora, México. 
es asintomático, y mujeres, quienes llegan a desarrollar inflamaciones del tracto reproductivo. De más de 100 tipos de virus que existen, 15 serotipos ${ }^{5}$ se consideran de alto riesgo, ${ }^{*, 3}$ y dos de éstos (16 y 18) originan 70\% de los casos de $\mathrm{CaCu}{ }^{6}$

El CaCu es la segunda causa de muerte de mujeres mayores de 25 años en México y Sonora. Los programas de detección oportuna de América Latina y el Caribe (a excepción de Cuba) han tenido un éxito limitado al mantener las tasas de incidencia y mortalidad más altas del mundo, sólo superadas por las de África Oriental y Melanesia. ${ }^{7}$ Se han registrado avances significativos en la investigación biomédica y epidemiológica sobre la forma de trasmisión del virus y su relación con el desarrollo del $\mathrm{CaCu}$, y se ha logrado una vacuna contra cuatro serotipos del virus. Pero es necesario indagar la dimensión sociocultural de estos problemas y su inclusión en la formulación de la política y promoción de la salud, la calidad de la atención y el fomento de la adherencia de las pacientes a los procesos de cuidado.

El objetivo es presentar un análisis relacional de cómo las mujeres con diagnósticos de VPH, displasia cervical y $\mathrm{CaCu}$ reciben y/o acceden a la información sobre estos problemas, y cómo ellas los viven y manejan en sus relaciones cercanas.

\section{Material y métodos}

De enero a junio de 2008 se entrevistó a 34 mujeres con una guía semiestructurada de preguntas abiertas, dando libertad a las entrevistadas de expresar preocupaciones, sentimientos, creencias y su percepción sobre el problema. Se utilizó el programa de apoyo al análisis cualitativo NVivo $7^{\ddagger}$ para la codificación de la información, organizada en seis nodos temáticos; uno de ellos referido a la información sobre $\mathrm{VPH}$, displasias y $\mathrm{CaCu}$, que recibieron las mujeres de los médicos y de sus redes cercanas.

Las mujeres fueron contactadas a través de dos clínicas de colposcopía de dos hospitales de la Secretaría de Salud en Sonora. A estas clínicas se canalizan mujeres con un diagnóstico de infección por VPH, displasia o $\mathrm{CaCu}$ in situ en los servicios de primer nivel de atención de Hermosillo, áreas rurales y centros de salud de otros municipios de Sonora.

\footnotetext{
* Se entiende por VPH de alto riesgo oncológico o VPH-AR a los virus asociados con procesos agresivos y acelerados de transformación celular vinculados principalmente a los serotipos $16,18,45,31,33$, $52,58,35,59,56,39,51,73,68$ y 66 .

₹ Nvivo 7 es un software de apoyo para análisis cualitativo desarrollado por QSR International Pty Ltd 2007.
}

Los criterios de selección de las informantes fueron: 1) pertenecer al grupo de edad de 25 a 45 años; 2) tener un diagnóstico de infección por VPH, displasia o $\mathrm{CaCu}$ in situ; 3) acudir a estas clínicas de colposcopía. El contacto se hizo a través del médico o enfermera de la clínica de colposcopía y 100\% de las mujeres aceptó participar. Se les explicó el consentimiento informado para su participación y para audiograbar la entrevista, utilizando seudónimos para proteger su confidencialidad. El protocolo de investigación y el consentimiento fueron aprobados previamente por el comité de ética de la institución.

Las mujeres se eligieron por diagnóstico y edad para tener una variabilidad equilibrada entre los diversos casos: las que estaban al inicio, a la mitad o al final del proceso de atención (cuadro I). Así se conformó una muestra de conveniencia, no representativa ni aleatoria.

El análisis se basó en la metodología de la teoría fundamentada, que permite construir conceptos y categorías de forma sistemática, con el objetivo de que la construcción teórica se ancle a la información empírica ${ }^{8}$ y relaciona las categorías con su marco contextual al explicar sus propiedades y dimensiones.

\section{Resultados}

En las trayectorias de atención de las mujeres diagnosticadas con $\mathrm{VPH}$, displasias o $\mathrm{CaCu}$ in situ se expresan procesos netamente socioculturales y con una alta dosis de contenido simbólico, al tratarse de una enfermedad aún posicionada en el imaginario colectivo con consecuencias fatales.

La mayoría de las mujeres declararon tener una práctica regular del Papanicolaou (Pap), que va de los 6 meses a los 3 años, por lo que consideramos que las entrevistadas tienen cierta información de las implicaciones y resultados de la prueba.

En las narrativas sobresale el tema del acceso y manejo de la información y su vinculación a creencias, valores y prejuicios. Sin embargo, no es una práctica sostenida en los servicios de salud brindar información accesible y oportuna a las pacientes, como tampoco es exigida por las mismas. En otra investigación se observó que la información ofrecida a las pacientes en la consulta de ginecología era muy escueta y con términos médicos, ${ }^{9}$ a pesar de que existe la obligación legal y ética de los médicos de dar información. ${ }^{10}$ Las mujeres no sabían que podían exigir explicaciones oportunas y sencillas sobre su problema y para orientar su gestión de la atención. Esto origina una agencia limitada y el hecho de depender en mayor medida de la discrecionalidad o casuística en los servicios de salud. Enseguida 
Cuadro I

Características sociodemográficas y atención médica de las mujeres diagnosticadas con VPH, DispLASIA del cuello del útero y Cáncer Cervicouterino. Hermosillo, Sonora, octubre de 2007 a octubre de 2008*

Grupos de edad

\begin{tabular}{lr}
$25-35$ años & 15 \\
\hline $36-45$ años & 19 \\
Escolaridad & \\
Ninguna & 1 \\
\hline Primaria & 5 \\
\hline Secundaria & 19 \\
\hline Preparatoria & 5 \\
\hline Universidad & 3 \\
\hline Sin dato & 1
\end{tabular}

Servicio de salud

\begin{tabular}{lr} 
Secretaría de Salud & 17 \\
\hline IMSS & 1 \\
\hline ISSSTESON & 3 \\
\hline Seguro Popular & 13
\end{tabular}

Frecuencia de la práctica del Pap

\begin{tabular}{lr} 
Primera ocasión & 4 \\
\hline De 6 meses a un año & 18 \\
\hline De 2 a 3 años & 6 \\
\hline Más de 3 años & 5 \\
\hline Sin dato & I
\end{tabular}

Diagnóstico

\begin{tabular}{lr} 
VPH & 2 \\
\hline Displasia leve & 13 \\
\hline Displasia leve $+\mathrm{VPH}$ & 3 \\
\hline Displasia moderada & $\mathrm{I}$ \\
\hline Displasia moderada $+\mathrm{VPH}$ & $\mathrm{I}$ \\
\hline Displasia severa & 4 \\
\hline Displasia severa $+\mathrm{VPH}$ & 4 \\
\hline CaCu in situ & 6
\end{tabular}

Etapa de la trayectoria

\begin{tabular}{lr} 
Diagnóstico & 7 \\
\hline Tratamiento & 5 \\
\hline Control & 14 \\
\hline Alta & 8
\end{tabular}

Tipos de intervenciones

\begin{tabular}{lr} 
Criocirugía & 8 \\
\hline Conización & 16 \\
\hline Histerectomía & 6
\end{tabular}

se presentan las categorías analíticas sobre el acceso y manejo de información y cómo las mujeres lo viven en sus relaciones cercanas. En cada testimonio, se anota la edad y el diagnóstico de la entrevistada.

\section{Información recibida/obtenida de los prestadores de salud}

Cuando las mujeres describieron sus trayectorias de atención, la relación médico-paciente cobró suma importancia, ya que muchas de sus experiencias y sentimientos de temor y angustia ante los diagnósticos estuvieron ligados al trato e información otorgada u omitida por los médicos. Mónica y Dora cuentan:

Vinieron a avisar...Como yo trabajaba, al otro día fui (al centro de salud). “QQué pasó?” le dije. Era un enfermero: espéreme tantito, no se mueva de aquí. Va por la enfermera y ella me dice: espérese aquí, quédate aquí. -¡Ay! ¿Qué podrá ser? Más me asustaba (hace gestos de cara asustada), porque me llevó a un cuartito, sola, y cerró la puerta, y ya me dijo que traía principios de cáncer... pero me dijo la enfermera que estaba a tiempo, que se podía curar. (31: displasia severa).

Todos esos días yo tuve mucho miedo a tener cáncer. Ese fue el miedo, que me saliera displasia... (42: displasia severa+VPH).

Ambas tenían una idea vaga de qué era una displasia y su asociación con el $\mathrm{CaCu}$; al recibir estos diagnósticos las mujeres expresaron su temor a la muerte, hecho que ha sido encontrado en otros estudios. , $^{11-13}$ En el imaginario social esto es una idea cultural fuertemente asentada, a pesar de la difusión sobre la detección temprana del $\mathrm{CaCu}$ y aun en mujeres con una práctica regular del Pap, como Lucía señala:

Yo me lo hago cada año... Cuando me hablaron pensé: ¿y ora?, si cada año me lo hago, pues ¿qué pasó?, ¿cómo es 
que de un año para otro ya traigo algo?, ¿por qué me iban a hablar? Pues porque era cáncer (32: displasia leve).

Otras mujeres, aunque manejaron la misma idea de fatalidad, términos como displasia y VPH eran desconocidos para ellas. Marta comenta:

(El) doctor me dijo: lo que pasa es que a usted le salió displasia. Pero yo en ese momento no sabía, ¡no!, es más, yo no conocía ni la palabra (45: displasia leve).

Se observa la angustia que genera el desconocimiento sobre el diagnóstico ante la ausencia de información accesible y oportuna por parte de los prestadores. Las experiencias alimentan la percepción de las mujeres sobre los médicos en sentidos contradictorios: por un lado, la confianza para autorizar las intervenciones, pero por otro, desconfianza de que no se les ofrece mayor información por la gravedad de su problema. Emilia, que fue histerectomizada, narra:

El caso es que cuando me operaron... el pleito mío con el doctor fue: ¿por qué a mí no me enseñó? ¿Por qué yo era a la única que no habían enseñado? ¿Por qué?, ¡porque me estoy muriendo, por eso! (45: $\mathrm{CaCu}$ in situ).

Lo expresado por las entrevistadas deja en evidencia la relación jerárquica y hegemónica entre médico-paciente, que pocas veces se transgrede por las pacientes. Iris señala:

Pues la verdad yo no sabía qué era la displasia leve..., nunca había pasado por nada de eso. Primero lloré; sí, me alarmé..., pensé: jay, a lo mejorar es cáncer o algo así! En el (hospital) no me han dicho nada..., el especialista no ha hablado bien conmigo..., o sea, si ha habido buena atención y todo, pero bien, bien, no me ha hablado del tema..., como yo quisiera, no (31: displasia leve).

Es primordial la valoración que las mujeres dan a la información que el médico les proporciona. En otros estudios se ha reportado que los pacientes desearían obtener información sobre su salud por parte de médicos y enfermeras. ${ }^{14}$ Ante la falta de información, algunas mujeres hicieron uso del Internet, pero no les resolvió totalmente la necesidad y entendimiento de la información, y en algunos casos, aumentó la angustia y desconfianza de las mujeres. Emilia y América contaron:

Cuando me diagnostican cáncer le digo a Lupita (su hija): quiero que entres al Internet..., quiero que veas qué significa carcinoma. $\mathrm{Y}$ ahí me está trayendo hojas y yo leyendo (45: $\mathrm{CaCu}$ in situ).
Él (médico) tenía unas fotos, y me dijo: mira, este grado tienes tú y así se ve, blanquizco. ¡Ah!, me quedé yo..., no le pregunté nada, él nomás explicaba. Dudas que yo tenía, de por qué es o por qué pasaba eso, lo busqué en el Internet (38: displasia severa +VPH).

La interacción jerárquica entre el médico y la paciente, mediada por el discurso dominante de la medicina y por las desigualdades de género, ${ }^{15,16}$ limita que las pacientes pregunten sus dudas y genera desconfianza, como lo expresó Emilia. Estudios reportan que las pacientes evalúan la comunicación con el médico en términos de su experiencia en el tema, confianza y respeto de su autonomía. ${ }^{17}$ Los pacientes que tienen información sobre su estado de salud muestran menores niveles de ansiedad, así como una mejor adherencia al tratamiento. ${ }^{18,19}$

\section{Vivencia del diagnóstico y familia}

Se identificó que las redes familiares y sociales ofrecen apoyo en los procesos de atención de las mujeres, pero el manejo de la información es limitado y reafirma los temores sobre el $\mathrm{CaCu}$.

Las redes de apoyo pueden ser sociales, comunitarias, familiares e institucionales y permiten que ante una crisis, como el diagnóstico de una enfermedad, las personas puedan mantener su equilibrio físico, emocional y social. ${ }^{20}$ Las redes brindan apoyo en la gestión, el cuidado postoperatorio y búsqueda de información, aunque ésta sea confusa, como lo narran Alondra y Telma:

Se me desguanzaron las piernas y lueguito empecé a sudar porque soy muy nerviosa y dije: pues tengo cáncer. Y luego luego le hablé a mi mamá y le dije: amá, habló la doctora. Dice que tengo displasia leve. Pues es cáncer, me dice... Me mandó pa'llá (clínica de colposcopía), me dijo del papiloma humano y de las vacunas... Pues bien asustada, ¡yo no hallaba qué hacer! (35: displasia leve+VPH).

Pues cuando yo necesité el apoyo fue de mi mamá, y cuando me operaron fue mi hermana mayor la que estuvo conmigo. Y ya cuando salí, días me miraba ella o mi mamá, y luego yo sola (37: displasia severa).

Los datos concuerdan con los de otros estudios que identifican el papel de la familia, y específicamente de la madre como encargada de transmitir la información sobre el autocuidado de la salud femenina, ${ }^{14}$ además de participar en el proceso de gestión, tratamiento y recuperación de la paciente. 
Pocas mujeres dijeron conocer o tener información sobre la infección por VPH y comparten algunas ideas del imaginario social, asignándole una connotación de estigma al considerar que se genera por promiscuidad e infidelidad en las parejas, hecho que limita la búsqueda de apoyo en las redes familiares. Isabel cuenta:

Realmente nadie sabe (sobre el diagnóstico), no les he dicho...; ellos (la familia) saben que vengo porque tengo una infección y posiblemente iba a tener cáncer. Yo nunca les dije..., porque no quiero que me juzguen, ni a él ni a mí, eso es todo..., es que son las más duras, la familia..., pues la familia de él dice que porque yo he tenido muchas relaciones sexuales con varios, no nomás él. Mi familia pues... va a decir lo mismo (38: displasia leve+VPH).

\section{Vivencia del diagnóstico y pareja}

En el estudio se identificó que los problemas por VPH, displasia o $\mathrm{CaCu}$ influyen de manera diferencial entre las mujeres y su impacto va cambiando a medida que se rebasa o se complica con otros asuntos de sus vidas, además de que se comparte la percepción de una relación indisoluble entre estos diagnósticos y su vida sexual. Sin embargo, el tema es poco tratado por los médicos al atenderlas. Si bien algunas comprendieron que la infección por VPH puede estar latente durante muchos años, y que no necesariamente se generó por alguna infidelidad de su pareja, su desconfianza persiste. Algunas de las entrevistadas sufrieron episodios de franco enojo y tomaron medidas que afectaron su vida de pareja. Eva e Isabel expresan:

Pues me sentía mal, ¿por qué?... no sé, no sé ni cómo explicarte, porque cuando uno no es así, pues se siente mal, luego dices: ¡ay, ay!, ¿por qué me volví a casar?, ¿ya estoy mal o qué? Por eso yo nomás me quedaba seria (frente al médico). Vine y le dije a mi esposo: ¡oye tú!... ¡veme la cara! ¿De qué la tengo?, ¿por qué?, me contestó. Es que me preguntó el doctor que cuántas parejas había tenido (35: displasia severa+VPH).

(El VPH) sé que es de transmisión sexual y que el hombre es el portador nomás..., pero uno no lo porta, no se lo contagia a otra persona, más que lo tiene allí uno..., no quiero que mis hijos se den cuenta porque..., el coraje que traigo..., jno puedo!, ya no puedo..., deseo ser fuerte (Isabel llora), ...el coraje, pues contra mi esposo, ¿contra quién más?... Aunque sé que me voy a curar, pero de todas maneras no puedo, el coraje ahorita es mucho... (38: displasia leve+VPH).
Algunas informantes preguntaron al médico sobre la necesidad de incorporar a su pareja al tratamiento, siendo una infección de trasmisión sexual, pero sus médicos tratantes desestimaron dicha atención, lo que incrementó la frustración y desconfianza sobre el médico y sus parejas. Natalia cuenta:

\footnotetext{
A mi marido no se le citó para nada, no me dijeron "dígale que se tome estas pastillas, que se ponga estas pomadas, que venga porque le vamos a hacer estos estudios". ¡Nada!..., aquí uno, porque uno es la que se puede morir, no el hombre. ¡Pues sí!... uno es la que se puede morir..., pero ¿cómo va a estar pasando cada vez esos procesos?, ¡si son lastimosos! (37: VPH).
}

En otros estudios se ha informado que los médicos contribuyen a los contenidos morales asignados a las enfermedades, especialmente aquéllas relacionadas con la sexualidad y el cuerpo de las mujeres. $15,16,21,22 \mathrm{En}$ este estudio las mujeres mencionaron que el tema de la sexualidad es poco abordado por los médicos. Además, la desinformación sobre el VPH y el estigma sobre el manejo de la vida sexual son factores que potencian otros problemas de pareja, al combinarse con otras creencias alrededor del problema. Ximena narra:

Él en nada me ayudó. Al contrario, me echó en cara que quién me pegó la enfermedad. Le dije: si tú sabes perfectamente que yo nomás con mi esposo; yo nunca, nunca he tenido otra pareja. Le dije: hazte el estudio. Pero supuestamente a él no se lo quisieron hacer..., ya cuando andábamos en el divorcio, con tal de ofender a uno... (38: displasia severa).

\section{Discusión}

Existe una constante necesidad de las mujeres pacientes de acceder, recibir y entender la información sobre la infección por $\mathrm{VPH}$, displasias y $\mathrm{CaCu}$ in situ que les permita disminuir sus estados de angustia y percepción de fatalidad que todavía se le asigna al $\mathrm{CaCu}$. Las pacientes no preguntan ni exigen las explicaciones al médico sobre su problema, ya que la relación desigual las cohíbe. ${ }^{9,15,23}$ Recibir información oportuna y accesible de los médicos permitiría a las mujeres apropiarse de sus procesos de atención. Aunque es una disposición legal y ética, los prestadores de salud no ofrecen información, lo que genera incertidumbre ante procesos desconocidos y obstaculiza una adecuada toma de decisiones, que se complica con componentes emocionales y valorativos. $11,17,24$ 
El manejo estigmatizante, como lo expresaron varias entrevistadas, limita buscar ayuda en sus redes, o bien, solicitar información, por el temor de sentirse señaladas, lo que demuestra cómo el ejercicio de la sexualidad femenina se halla cargado de mitos y sometido al juicio social, percepción que se refuerza al ser un asunto poco tratado por los médicos. Mientras estos problemas sean desconocidos por la población lega, la analogía entre $\mathrm{VPH}$-displasia- $\mathrm{CaCu}=$ muerte será común en el imaginario social, como se ha confirmado en las entrevistas analizadas.

El personal médico debe capacitarse para reconocer las necesidades de información de las pacientes y las actitudes de angustia y desconfianza en las mismas. ${ }^{19}$ Debe hacerse visible la relación jerárquica entre el médico y las pacientes, así como las desigualdades de género inmersas en tal relación, para crear canales efectivos de comunicación.

\section{Referencias}

I. Walboomers J, Jacobs M, Manos M, Bosch X, Kummer A, Shah K et al. Human Papillomavirus is a necessary cause of invasive cervical cancer worldwide.J Pathol 1999; 189:13-19

2. Cutts F, Franceschi S, Goldie S, Castellsague X, de Sanjose S, Garnett G et al. Human papillomavirus and HPV vaccines: a review. Bull World Health Organ 2007; 85:719-726.

3. Hernández-Hernández D, Apresa-García T,Alvarado-Cabrera I, GarcíaCarrancá A, Guido-Jiménez M, González-Sánchez J et al. Virus de papiloma humano de alto riesgo (VPH-AR) y neoplasia intraepitelial cervical (NIC) en mujeres de dos hospitales de la Ciudad de México. Rev Invest Clin 2002; 54:299-306.

4.Tirado-Gómez L, Mohar-Betancourt A, López-Cervantes M, GarcíaCarrancá A, Franco-Marina F, Borges G. Factores de riesgo de cáncer cervicouterino invasor en mujeres mexicanas. Salud Publica Mex 2005; 47:342-350.

5. Muñoz N, Bosch X, Castellsague X, Diaz M, de Sanjosé S, Hammouda $D$, et al. Against wich human papillomavirus types shall we vaccinnate and screen? The international perspective. Int J Cancer 2004; I I I:278-285 6. Bosch FX, Castellsagué $X$, de Sanjosé, S. HVP and cervical cancer: screening or vaccination? Br J Cancer 2008; 98:I5-2I.

7. Organización Panamericana de la Salud, Unidad de Género, Etnia y Salud. Indicadores básicos para el análisis de la equidad de género en salud.Washington: Organización Panamericana de la Salud, 2004. 8. Strauss A, Corbin J. Bases de la investigación cualitativa. Técnicas y procedimientos para desarrollar teoría fundamentada. Colombia: Universidad de Antioquia, 2002.
9. Castro M. De pacientes a exigentes. Un estudio sociológico sobre la calidad de la atención, derechos y ciudadanía en salud. Hermosillo: El Colegio de Sonora, 2008.

10. Comisión Nacional de Arbitraje Médico [sitio de internet]. México: CONAMED; c 2007. CONAMED Carta de los derechos de los pacientes; [aprox. I0 pantallas]. [Consultado 2009 ago 20]. Disponible en: http:// www.conamed.gob.mx/interiores.php?ruta=http://www.conamed.gob.mx/ publicaciones $/$ cartas $/$ \&destino $=$ cartapacientes. php\&seccion $=22$

II. Weisner C, Vejarano M, Caicedo J, Tovar S, Cendales R. La citología del cuello uterino en Soacha, Colombia: representaciones sociales, barreras y motivaciones. Rev Salud Pública 2006; 8: 185-196.

12. Cartwright E, Salazar G, Castro MC. "Soy muy mujer". Percepciones del cáncer cervicouterino en mujeres mayores: Hermosillo, Sonora, y Tucson, Arizona. En: Denman C, Monk J, Ojeda de la Peña N, ed. Compartiendo historias de fronteras: cuerpos, géneros, generaciones y salud. México: El Colegio de Sonora, 2004:181-209.

13. Reartes-Peñafiel D. La infección por el virus del papiloma humano (VPH): conflictividades, miedos y ambivalencias. Un estudio con parejas y médicos en un hospital del 3er. Nivel de atención de la Ciudad de México (tesis). Guadalajara: Centro de Investigaciones y Estudios Superiores en Antropología Social, 2005.

14. Stewart D,Abbey S, Shnek Z, Irvine J, Grace S. Gender differences in health information needs and decisional preferences in patients recovering from an acute ischemic coronary event. Psychosom Med 2004; 66:42-48. 15. Turner B. Medical power and social knowledge. Londres: SAGE Publications, 1995.

16. Freidson E. La profesión médica. Barcelona: Península, 1970. 17. Burkitt E, Holcombe Ch, Salmon P. Doctors' communication of trust, care and respect in breast cancer: qualitative study. BMJ 2004. 18. Barnato A, Llewellyn-Thomas H, Peters E, Siminoff L, Collins D, Barry M. Communication and decision making in cancer care: setting research priorities for decision support/patients' decision aids. Med Decis Making 2007; 27:626-634.

19. Leydon G, Boulton M, Moynihan C, Jones A, Mossman J, Boudioni M, et al. Cancer patients' information needs and information seeking behaviour: in depth interview study. BMJ 2000; 320:909-913.

20. Guzmán J, Huenchuan S, Montes de Oca V. Redes de apoyo social de personas mayores: marco teórico conceptual. Ponencia presentada en Simposio Viejos y Viejas. Participación, ciudadanía e inclusión social. 51 Congreso Internacional de Americanistas; 2003 jul I4-18; Santiago de Chile.

21. García S, Becker D, Tatum C,Aldrich T, Fernández A. Linking cervical cancer to the human papillomavirus: findings from a qualitative study with mexican women. Health Care Women Int 2007; 28: 192-205.

22. Hunt L. Moral reasoning and the meaning of cancer: causal explanations of oncologist and patients in southern Mexico. Med Anthropol Q 1998; 12:298-318.

23. Waitzkin H.The politics of medical encounters. How patients and doctors deal with social problems. New York:Yale University Press, 1991. 24. Da Silva Marques-Ferreira M, Gimenez-Galvao M. Percepción de un grupo de mujeres acerca del hecho de ser portadoras delVPH. Ginecol Obstet Mex 2005; 73:531-536. 